\title{
Perioperative Considerations: Planning, Intraoperative and Postoperative Management
}

\author{
Leo F. S. Ditzel Filho - Daniel M. Prevedello • \\ Mihir R. Patel - Bradley A. Otto · Ricardo L. Carrau
}

Published online: 19 October 2013

(C) Springer Science+Business Media New York 2013

\begin{abstract}
Throughout the last decade, the use of endoscopic skull base surgery has increased, aggregating the efforts of otolaryngologists-head and neck surgeons, and neurosurgeons. Using the natural sinonasal corridor to reach virtually the entire ventral skull base, this aspect of skull base surgery has grown exponentially, adding anatomy-based modular techniques that enable the safe removal of complex neoplasms, even in the presence of intradural progression or vascular encasement. Nonetheless, the safe employment of this technique requires fastidious planning, meticulous intraoperative management and thorough postoperative care to yield optimal results and prevent complications. Patients are preoperatively submitted to a clinical evaluation and a series of complementary imaging studies in order to warrant proper selection of the technique. During surgery, appropriate training and adequate resources, coupled with skilled anesthesiology support, allow the surgical team to safely perform the transnasal approach, dissection and resection of the tumor and reconstruction of the resultant defect. The postoperative period is equally important and aims not only to facilitate a speedy recovery, but also to prevent potential complications. This study describes those measures in the planning and perioperative management of skull base lesions through endonasal surgery, as used by our group.
\end{abstract}

L. F. S. Ditzel Filho · D. M. Prevedello ( $₫)$

Department of Neurological Surgery, The Ohio State University

Wexner Medical Center, N-1049 Doan Hall, 410 W. 10th

Avenue, Columbus, OH 43210, USA

e-mail: daniel.prevedello@ osumc.edu

M. R. Patel - B. A. Otto - R. L. Carrau

Department of Otolaryngology - Head and Neck Surgery,

The Ohio State University Wexner Medical Center, Columbus,

$\mathrm{OH}$, USA
Keywords Endonasal - Endoscopic $\cdot$ Skull base $\cdot$ Skull base surgery $\cdot$ Skull base tumor - Perioperative management $\cdot$ Transnasal

\section{Introduction}

Over the last decade, otolaryngologists and neurosurgeons have witnessed the rise and growing popularity of endoscopic skull base surgery. This relatively new subspecialty has brought the two fields together, spawning a wealth of new approaches, instruments and dedicated literature [1-10]. At its current state, there are very few contraindications to endonasal approaches to the skull base, and these are generally dictated by patient and pathology characteristics [11], surgeon's experience and training, as well as the availability of adequate resources. Lesions that expand beyond the orbital meridian and sinonasal tumors that infiltrate the orbital soft tissues or the facial skin are examples of clinical scenarios in which an "open" approach is most beneficial for the patient.

Once a patient has been deemed suitable for an endonasal approach, a series of steps are taken to properly plan and safely execute the procedure. This review will detail the measures involved in the surgical planning and the intra- and postoperative management of these patients.

\section{Planning}

All patients undergoing endonasal approaches are referred for preoperative assessment by anesthesiology. At this consult their nostrils are swabbed for methicillin-resistant (MRSA) and methicillin-sensitive Staphylococcus aureus (MSSA). When the patient's culture grows MRSA, we 
prescribe mupirocin ointment to be applied on the nostrils twice daily for 5 days preceding surgery. During induction, these patients will also receive a combination of perioperative prophylactic antibiotics that includes intravenous vancomycin. A negative culture requires no further measure.

Preoperative planning relies heavily on imaging [12], coupled with the patient's symptoms. All patients undergo thin-cut $(1.0-\mathrm{mm})$ contrasted magnetic resonance imaging (MRI) scans and a computerized tomography (CT), or computerized tomographic angiography (CTA) for planning and intraoperative image guidance. The surgical team, comprised of an otolaryngologist and a neurosurgeon, meticulously analyzes the scans to plan resection strategies and to anticipate possible complications [13].

MRI provides exquisite soft tissue definition, which is especially useful in detecting dural involvement, brain or orbital invasion and perineural tumor spread, as well as distinguishing tumor in the sinonasal tract from trapped secretions or edematous mucosa. Conversely, a thin-cut CTA is helpful in identifying bone infiltration and erosion as well as the relationship between the vasculature and tumor. Therefore, we perform a CTA in all patients selected for an expanded approach and those undergoing reoperations. These studies are uploaded and fused into an optical intraoperative navigation device (Stryker Navigation; Kalamazoo, MI, USA). This combination increases the accuracy of the navigation device and enables the surgeon to perform intraoperative bone refinement, utilizing the bony surfaces of the skull base to add reference points. Interventional angiography and/or embolization is not typically used, with the exception of highly vascularized extradural lesions such as giant juvenile nasopharyngeal angiofibromas (JNAs). In situations where the tumor has completely encased the ICA or significantly compressed the vessel's lumen, an angiography with balloon test occlusion is indicated. A xenon CT or a single-photon emission CT (SPECT) follows the balloon test occlusion to determine whether the cerebrovascular collateral system is sufficient to provide an adequate cerebral blood supply in case that one encounters the rare instance of needing sacrifice of the ICA. If the patient fails the test (i.e., the xenon CT or SPECT detects areas of hypoperfusion after carotid occlusion), a vascular bypass or endovascular stent should be considered.

A preoperative biopsy should be performed on all accessible tumors and can be completed in the office in select cases. However, most intradural and deep bony lesions are not easily amenable to preoperative biopsies (i.e., a biopsy would require an approach that is similar to that of the resection); thus, intraoperative histological analysis (i.e., frozen sections) is requested during the main procedure. Patients harboring malignancies should also be evaluated for systemic disease, typically through a positron emission tomography (PET). If drop metastases are suspected (e.g., high grade sarcomas), a complete spine MRI and lumbar puncture for cerebrospinal fluid (CSF) cytology evaluation are indicated.

Imaging aids the team in determining the goals of surgery. In the majority of instances, the aim is a complete resection of the lesion; however, specific conditions may not be amenable to radical resection, or its total removal may infer significant sacrifice of function (e.g., neurological deficit). For example, when dealing with benign tumors such as cavernous sinus meningiomas, the surgeons may opt to perform a decompression and subtotal resection to preserve cranial nerve function. In addition, when approaching sinonasal malignancies with disseminated metastasis or those with a local invasion that does not allow a complete surgical resection, surgery may aim for palliation, thus promoting a relief of symptoms and cytoreduction before adjuvant therapy is initiated. These cases warrant a thorough multidisciplinary discussion with all involved disciplines before surgery takes place. Lastly, very large tumors may be submitted to plan staged resection, with or without open approaches to maximize tumor resection. This is especially true in complex (multi-compartment, vascular-encasing) slow-growing lesions, such as some meningiomas and chondrosarcomas.

Patients presenting with visual changes due to tumor compression must be formally evaluated by ophthalmology prior to surgery, ideally with visual field testing. This establishes a baseline function for comparison and assesses the degree of severity of the visual impairment. Similarly, patients presenting with hearing loss must also be submitted to an audiogram. Following the same principle, if there is a plan for olfaction preservation on anterior skull base tumors, then this function should also be objectively assessed preoperatively. Lesions extending toward the jugular foramen mandate a voice and swallowing evaluation by a speech pathologist. In these cases, both the team and patient must be prepared for the need for a tracheotomy and/or a percutaneous endoscopic gastrostomy (PEG) tube, when significant lower cranial nerve deficits are observed or expected after surgery.

Patients with pituitary tumors or other lesions in the sellar and suprasellar regions must have their pituitary function thoroughly analyzed. A full pituitary panel (Table 1) should be obtained, and the endocrinology team must always be involved to evaluate for hypopituitarism or hormone overproduction. The patient must be questioned for signs of diabetes insipidus (DI), especially if the tumor displaces or even infiltrates the pituitary stalk, as commonly seen in craniopharyngiomas. In many instances, the patient is already suffering from a compensated DI, and desmopressin should be prescribed and adjusted to an 
Table 1 The pituitary panel: these are the hormones directly or indirectly produced by the pituitary gland that must be evaluated prior to endonasal surgery of the sellar and suprasellar regions
Pituitary panel:
1. ACTH
2. Cortisol
3. Growth hormone
4. Insulin-like growth factor 1 (IGF-1)
5. Prolactin
6. Thyroid-stimulating hormone (TSH)
7. Free triiodothyronine (T3)
8. Total thyroxine (T4)
9. Testosterone
10. Luteinizing hormone (LH)
11. Follicle-stimulating hormone (FSH)

appropriate dosage to prevent hypernatremia, particularly during the preoperative fasting period.

Another key aspect of preoperative planning concerns reconstruction tactics. A history of previous sinonasal infection or chronic sinusitis must be scrutinized as well as any previous history of sinonasal surgery or craniotomy. The workhorse of skull base reconstruction after expanded endonasal approaches (EEAs) is the pedicled nasoseptal flap (Hadad-Bassagaisteguy flap). Any suspicion that its vascular supply may have been compromised by previous surgical trauma and a history of radiation or infection requires a contingency plan. Intraoperative acoustic Doppler sonography assesses the flap viability [14]; nonetheless, the surgeon must be prepared to perform an alternative endonasal or regional reconstructive flap [15]. The possible need for additional autologous grafting, typically abdominal fat or fascia lata, must also be considered. The patient must be aware of their potential need, as well as the possibility that a fully endonasal reconstruction may need to be converted into an open procedure. A strong suspicion of infectious or inflammatory activity calls for an endoscopic office evaluation, and any abnormal findings must be addressed before surgery.

Finally, patients must be fully informed about the nature, extent and prognosis of their condition, the process they are about to undergo, as well as alternatives of treatment. A detailed explanation of the surgical goals, risks and postoperative sequelae avoids unrealistic expectations from both the patients and their families as well as from the surgical team. All patients must sign a consent form, which details the procedure and the possible risks and complications that may occur, especially related to CSF leakage, neurological deficits and anosmia. In addition, they must also be informed of the possible need for blood transfusion.

\section{Intraoperative Management}

\section{General Preparations}

On the day of surgery, the patient is transported to the operating room, where general anesthesia is induced in standard fashion, following neurosurgical principles of intracranial pressure control, therefore avoiding drugs such as halothane. Intraoperative monitoring needles are placed according to the patient's needs. Intraoperative neurophysiologic monitoring also impacts the choice of anesthetic, especially when direct monitoring electromyography (EMG) or cranial nerve stimulation is expected and the patient cannot be paralyzed. All patients are monitored for somatosensory evoked potential (SSEP), while cranial nerve EMG monitoring is performed in select patients as dictated by tumor location, i.e. lesions invading the cavernous sinus mandate monitoring of cranial nerves III, IV and VI, while lesions extending to the jugular foramen indicate the monitoring of cranial nerves IX, $\mathrm{X}$ and XI. Specialized nerve stimulators, shaped as dissectors (e.g., the Kartush ${ }^{\circledR}$ set, multiple suppliers available), are particularly useful in cases with tumor encasement.

Endonasal skull base surgery uses the natural corridor of the nasal cavity and sinuses to gain access to the ventral skull base; thus, the patient must receive adequate broad antibiotic coverage with activity against the local flora, including Haemophilus influenzae, Streptococcus pneumoniae and Branhamella catarrhalis. At our institution, all patients who will have transdural surgery receive a fourth generation cephalosporin (i.e., cefepime) upon anesthetic induction, provided they are not allergic. Patients allergic to cefepime (or to penicillins) receive aztreonam and vancomycin. If the patient's preoperative nasal swab detects MRSA, vancomycin is also administered, even after proper use of mupirocin. Any nasal packing requires further antibiotic coverage, typically sulfamethoxazoletrimethoprim, until the packing is removed (postoperative day 3-5).

Once anesthesia has been achieved, the endotracheal tube is positioned and secured toward the patient's left side, avoiding obstructing the surgeon. The eyes are lubricated, securely closed and protected with Tegaderm dressings (3M, St. Paul, MN, USA). A grounding paddle for the electrocautery is placed on the posterior surface of one of patient's thighs, and a Foley catheter is inserted not only to relieve the patient's bladder, but also to provide the anesthesiologist with invaluable data regarding urinary volume. This is especially important when the lesion displaces the pituitary stalk and intraoperative onset of DI is a possibility.

Deep vein thrombosis (DVT) prophylaxis is important; however, anticoagulation and platelet anti-aggregation therapy are contraindicated in transdural surgery because 
of the dire consequences of bleeding in this area. Therefore, the patient's legs are outfitted with sequential compression devices.

A central line is not mandatory; however, it is recommended during expanded approaches in which drilling around the ICAs or through the clivus is to take place. Similarly, it is prudent to prepare the patient for the use of blood products. Packed red blood cells should be typed and cross-matched to the patient when significant blood loss is anticipated. All patients undergoing expanded approaches are typed and cross-matched for two units of packed blood cells and cryoprecipitate. Use of cell saver devices in EEAs is limited by the potential contamination by the nasal flora, tumor cells and the difficult logistics associated with its use.

\section{Patient Positioning}

The patient is placed in supine position with the head secured on the Mayfield three-pin head fixator (Fig. 1a) when there is a need for EMG cranial nerve monitoring and the patient will not be paralyzed. Other relative indications to fix the head include the expectation of a prolonged surgery and the need for extensive drilling of the skull base. Otherwise, a horseshoe-type head-holder or conforming pillow is adequate to maintain the head in position. The neck is secured in a position that is slightly extended, tilted to the left and turned to the right. The patient's arms are secured parallel along the cranial-caudal axis, while the abdomen is maintained exposed (Fig. 1a). The patient's body is also secured to the operating table, thus, allowing right or left listing in order to improve the ergonomics of the surgery (Fig. 1b). In patients at high risk for an ICA injury, the right thigh is also prepared and exposed for potential muscle harvesting. The abdomen and thigh are prepped with chlorhexidine and the face and nostrils with povidone-iodine solution.

A registration mask (Stryker Corp., Kalamazoo, MI, USA) is placed on the patient's face and a navigation tracker is attached to the Mayfield clamp. Once the facemask registration is complete, the data are transferred, so the navigation continues with the fixed tracker and the mask is removed. In short surgeries with no need for intraoperative EMG monitoring, i.e., most surgeries for pituitary tumors, encephaloceles or CSF leaks, the patient's head will rest on a horseshoe head-holder, conforming pillow or gel "donut;" therefore, the navigation mask is kept and covered by a transparent sterile drape that allows the camera to detect the light-emitting diodes (LEDs).

\section{Surgical Considerations}

The nasal cavity is decongested with oxymetazoline hydrochloride (Afrin ${ }^{\circledR}$, Schering-Plough Health Care
Products, Kenilworth, NJ, USA) applied by spraying or the insertion of soaked cotton pledgets. The patient is then covered with standard sterile craniotomy drapes, centered on the patient's face and with the nostrils left exposed (Fig. 1c, d).

Adjuvant equipment includes:

- Two suction tubes

- Suction electrocautery

- Endonasal bipolar forceps (Storz Endoscopy, El Segundo, CA, USA)

- Endonasal microdebrider (Medtronic, Minneapolis, MN, USA)

- TPS Saber drill (Stryker Corporation, Kalamazoo, MI, USA), 4-mm hybrid burr

- Angled Stylus Midas Rex drill (Medtronic; Minneapolis, MN, USA), 4-mm coarse diamond burr

- Side-cutting aspiration device (Myriad ${ }^{\circledR}$, NICO Corporation, Indianapolis, IN, USA)

- Endonasal dissection instruments set (KLS Martin, Jacksonville, FL, USA)

All reconstructive needs should be anticipated and alternative plans considered. In general, sellar, spontaneous or iatrogenic CSF leaks may be reconstructed with free tissue grafts, whereas, extended approaches are best reconstructed by pedicled vascular flaps. The nasoseptal flap is our preferred option for most defects. However, when the nasoseptal flap is compromised, alternative reconstruction techniques are employed. These may require scalp incisions, as in the pericranial and temporoparietal fascia flaps; thus, draping and prepping must be adjusted accordingly. Assessment of the nasoseptal flap availability can be ascertained using an acoustic Doppler sonographic endonasal probe [14].

The otolaryngologist begins the approach and, when right-handed, will stand to the right of the patient. The neurosurgeon joins the field after or during the approach according to the complexity and needs of the case. With this in mind, two monitors are set at the patient's left close to each surgeon's eye level (Fig. 1e). If one surgeon is left handed, the two monitors are placed opposite to each other, at both sides of the patient's head. Regardless, the navigation tower is placed at the patient's head.

Unlike their transcranial counterparts, endonasal approaches do not require adjuvant methods of intracranial pressure control. The patient is maintained at normocapnia, and mannitol and other diuretics are not typically used. A Valsalva maneuver is only used to aid in the identification of discrete CSF leaks. Use of lumbar spinal drainage is restricted to those rare cases of postoperative CSF leaks that are taken back to the operating room for secondary skull base rerepair. Currently, lumbar spinal drains are not primarily inserted even in the presence of intraoperative 
Fig. 1 Patient positioning, draping and operative setup. a The patient is supine with the head secured on a Mayfield three-pin headholder and discrete neck extension; the face and abdomen have been prepped, and monitoring needles can be observed. b The head is slightly turned to the right, and the bed is discretely tilted to the left if necessary (observe the restraints along the patient's arms). c, d Sterile drapes are applied over the face and around the periumbilical region. e The surgeons are positioned along the patient's right; the surgical technician is across the field on the patient's $l e f t$; the navigation tower and monitors can also be seen at the edges of the surgical table
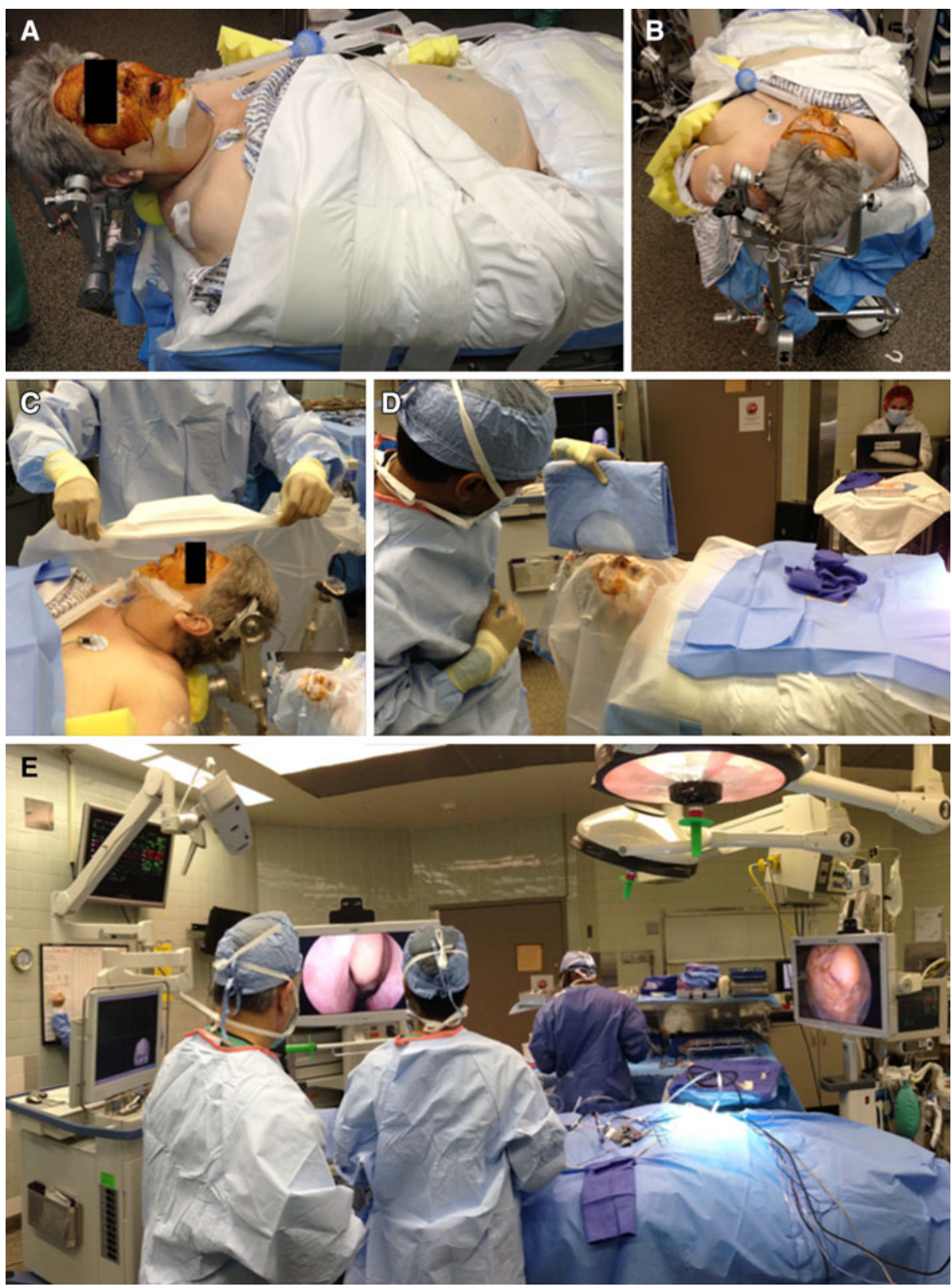

high-flow leaks such as those associated with craniopharyngiomas involving the third ventricle.

Patients with tumors transgressing the pia or with damage to the brain parenchyma by tumor compression or infiltration, as seen in large anterior cranial fossa meningiomas, are at risk for seizure activity. Therefore, levetiracetam is initiated intraoperatively and continued for a few days as prophylaxis.

Patients with pituitary adenoma in general do not receive corticosteroids during surgery to avoid adrenocorticotropic hormone (ACTH) suppression and false postoperative hypocortisolism. In patients with Cushing's disease, intraoperative ACTH levels are drawn during and after tumor dissection. Similarly, in cases of acromegaly or gigantism, growth hormone (GH) levels are measured during and after tumor dissection. Patients with suprasellar lesions, especially craniopharyngiomas, have their sodium levels and urinary output closely monitored.

All tumors are sent to pathology for intraoperative histological analysis (frozen sections); in all tumors with bone involvement (i.e., chordomas, chondrosarcomas and meningiomas), we sample the skull base for permanent analysis. Upon completion of the tumor resection, reconstruction of the skull base defect ensues. As previously mentioned, a variety of methods and techniques can be used to isolate the intradural space from the sinonasal cavity. Each method is tailored to the type and size of the defect while taking into account the patient's regional 
anatomy [16•]. Large defects are generally reconstructed with vascularized tissue, such as a pedicled nasoseptal flap. The reconstructive tissue is then bolstered by non-adherent material followed by expandable-sponge nasal packing, the balloon of a Foley catheter or any other suitable supportive material.

If the entire surgical resection is performed extradurally without CSF exposure, there is no need for reconstruction in most circumstances. An exposed ICA, however, requires coverage to avoid desiccation and to prevent rupture, particularly after radiation treatment. A nasoseptal flap provides adequate coverage. Minimal CSF transudation ("weeping") after pituitary surgery is frequent and can be managed with free tissue grafting (i.e., mucoperiosteum from the middle turbinate or abdominal fat).

When surgery is complete, the patient is awakened from anesthesia and extubated. Special care is taken by the anesthesiology team during this portion of the procedure to prevent excessive coughing and gagging, which may displace the reconstruction materials and thus cause a CSF leak. It is also important to communicate the contraindication for the passage of nasal tubes (e.g., nasogastric tube) and positive-pressure mask ventilation.

\section{Postoperative management}

After surgery, the patient is taken to either the post-anesthesia recovery room or an intensive or critical care unit (ICU or CCU), according to the complexity of the case. In general, pituitary tumor patients are sent to an intermediate level of care unit for the first 24 postoperative hours, where frequent neurologic, electrolytic and fluid balance assessments can be performed. The majority of patients undergoing an expanded approach are sent to the ICU, especially if there is dural transgression and subsequent manipulation of neural tissue. All others, including those undergoing surgery to repair CSF leak and patients with meningoceles or encephaloceles, are sent to a regular post-surgical bedroom after a brief period in the post anesthesia recovery area.

All patients are submitted to a non-contrasted head CT immediately after surgery to assess possible complications, particularly pneumocephalus. Oncologic patients are also submitted to a non-contrasted and contrasted MRI to evaluate the adequacy of resection. Typically this is completed during the first postoperative $24 \mathrm{~h}$ to avoid inflammatory artifacts. This MRI objectively establishes the degree of resection; therefore, contributing critical information for the decision-making process regarding the need for adjuvant therapies and serving as a baseline for future comparison. In addition, it also confirms the viability and correct positioning of the reconstructive material.
Hematology and serology studies (to ascertain fluid, electrolyte, acid-base balance and hemoglobin and white blood cell counts) are performed according to the needs of each patient and institutional protocols. Special attention is given to sodium levels and urine osmolality, which should be measured more frequently in patients with suprasellar lesions harboring an increased risk for DI, such as those with large craniopharyngiomas. If new-onset DI is suspected and the patient is awake and coherent, oral hydration is encouraged, and sodium levels are closely watched for the following 48-72 h. If DI persists, desmopressin supplementation is initiated.

All patients undergoing surgery for recurrent pituitary tumors have their cortisol and prolactin levels measured in the morning of the first postoperative day. Those with acromegaly and gigantism also have their GH levels assessed. Patients with Cushing's disease also have cortisol levels drawn every $6 \mathrm{~h}$ during the first $48 \mathrm{~h}$ after surgery, as well as an ACTH measurement in the morning of the first postoperative day. Corticosteroid supplementation is not employed unless the patient presents with signs of hypocortisolism preoperatively, or when it occurs postoperatively, which is often the case after a successful surgery for Cushing's disease.

Antibiotic prophylaxis is maintained during the first 48 postoperative hours, following the same regimen initiated in the operating room. If nasal packing is in place, then sulfamethoxazole-trimethoprim is prescribed until the packing is removed, traditionally on the third to fifth postoperative day.

Patients are evaluated daily for the possible presence of a CSF leak; if they report clear nasal drainage, they are challenged with a head tilting maneuver. In addition, an increase of pneumocephalus in a non-contrasted $\mathrm{CT}$ or direct bedside nasal endoscopy can confirm whether a leak is indeed present. Testing for beta- 2 or beta trace protein is specific and sensitive, but logistics dictate that these tests are not run daily in most institutions in the USA. CSF leakage is dealt with aggressively; all patients are taken back to the operating room for revision of their reconstruction as soon as possible. The vast majority of times, a leak is due to migration of the reconstructive tissue, allowing a fistula $[17 \bullet \bullet]$. To avoid this problem during the initial 6 weeks after surgery, all patients must refrain from blowing their nose and from activities that increase intracranial pressure, including squatting and bending over. The use of incentive spirometry is also restricted in the immediate postoperative period, and patients are advised to cough and sneeze only with their mouth open. Furthermore, use of stool softeners is also employed to avoid the need for abdominal muscle contraction during bowel movements. These measures, coupled with antibiotic prophylaxis, are responsible for the low rate of infectious complications observed in endonasal skull base surgery [18••]. 
All patients are encouraged to ambulate as soon as possible; until then, DVT prophylaxis continues in the form of sequential compression devices. Foley catheters are inserted for all tumor patients or when the estimated operative time is longer than $2 \mathrm{~h}$; these are typically removed in the morning of the first postoperative day.

Analgesia is obtained with mild narcotics to avoid altered levels of consciousness or even constipation. Nonsteroidal antiinflammatory drugs (NSAIDs) are avoided during the first week after surgery because of increased risk of nasal mucosal bleeding.

Nasal care is guided by the otorhinolaryngology team [19•]. Patients are encouraged to spray nasal saline solutions at least four times. An office visit is arranged for the first postoperative week for debridement and crusting removal. Further appointments are scheduled according to the degree of crusting and vary from patient to patient and between procedures performed (standard or expanded approaches).

\section{Conclusion}

Endoscopic endonasal surgery of the skull base is an expanding field that enables the surgeon to remove complex lesions through minimal access corridors. Nonetheless, fastidious planning, meticulous intraoperative management and thorough postoperative care are imperative to yield optimal results and prevent complications. It is paramount that surgeons pursuing endoscopic endonasal skull base surgery have proper training and understand the principles of rhinology, pituitary surgery, traditional skull base surgery and cerebrovascular surgery. Adequate equipment, institutional support and multidisciplinary cooperation are also essential to deliver proper treatment to patients with skull base pathologies and to avoid complications.

\section{Compliance with Ethics Guidelines}

Conflict of Interest Ricardo L. Carrau and Daniel M. Prevedello are consultants for Medtronic Corp. and have grants/grants pending with Storz Endoscopy, Stryker Corp., Medtronic Corp., KLS Corp., Vycor Medical Corp. and Integra Corp.; funding goes to their institution. Leo F.S. Ditzel Filho, Mihir R. Patel and Bradley A. Otto declare that they have no conflict of interest.

Human and Animal Rights and Informed Consent This article does not contain any studies with human or animal subjects performed by any of the authors.

\section{References}

Papers of particular interest, published recently, have been highlighted as:
- Of importance

-• Of major importance

1. Prevedello DM, Ditzel Filho LF, Solari D, Carrau RL, Kassam $\mathrm{AB}$. Expanded endonasal approaches to middle cranial fossa and posterior fossa tumors. Neurosurg Clin $\mathrm{N}$ Am. 2010;21(4):621-35 vi.

2. Prevedello DM, Pinheiro-Neto CD, Fernandez-Miranda JC, et al. Vidian nerve transposition for endoscopic endonasal middle fossa approaches. Neurosurgery. 2010;67(2 Suppl Operative):478-84.

3. Zada G, Agarwalla PK, Mukundan S, Dunn I, Golby AJ, Laws ER. The neurosurgical anatomy of the sphenoid sinus and sellar floor in endoscopic transsphenoidal surgery. J Neurosurg. 2011;114(5):1319-30.

4. de Lara D, Ditzel Filho LFS, Prevedello DM, Otto BA, Carrau RL. Application of image guidance in pituitary surgery. Surg Neurol Int. 2012;3(Suppl 2):S73-8.

5. Komotar RJ, Starke RM, Raper DM, Anand VK, Schwartz TH. Endoscopic endonasal compared with microscopic transsphenoidal and open transcranial resection of craniopharyngiomas. World Neurosurg. 2012;77(2):329-41.

6. Komotar RJ, Starke RM, Raper DM, Anand VK, Schwartz TH. Endoscopic endonasal versus open transcranial resection of anterior midline skull base meningiomas. World Neurosurg. 2012;77(5-6):713-24.

7. McLaughlin N, Eisenberg AA, Cohan P, Chaloner CB, Kelly DF. The value of endoscopy for maximizing tumor removal in endonasal transsphenoidal pituitary adenoma surgery. J Neurosurg. 2013;118(3):613-20.

8. McLaughlin N, Vandergrift A, Ditzel Filho LF, et al. Endonasal management of sellar arachnoid cysts: simple cyst obliteration technique. J Neurosurg. 2012;116(4):728-40.

9. Wang J, Bidari S, Inoue K, Yang H, Rhoton A Jr. Extensions of the sphenoid sinus: a new classification. Neurosurgery. 2010;66(4):797-816.

10. Caicedo-Granados E, Carrau R, Snyderman CH, et al. Reverse rotation flap for reconstruction of donor site after vascular pedicled nasoseptal flap in skull base surgery. Laryngoscope. 2010;120(8):1550-2.

11. Benet A, Prevedello DM, Carrau RL, et al. Comparative analysis of the transcranial "Far Lateral" and endoscopic endonasal "Far Medial" approaches: surgical anatomy and clinical illustration. World Neurosurg. 2013;. doi:10.1016/j.wneu.2013.01.091.

12. Grindle CR, Curry JM, Kang MD, Evans JJ, Rosen MR. Preoperative magnetic resonance imaging protocol for endoscopic cranial base image-guided surgery. Am J Otolaryngol. 2011;32(6):451-4.

13. Kasemsiri P, Solares CA, Carrau RL, et al. Endoscopic endonasal transpterygoid approaches: anatomical landmarks for planning the surgical corridor. Laryngoscope. 2013;123(4):811-5.

14. Pinheiro-Neto CD, Carrau RL, Prevedello DM, et al. Use of acoustic Doppler sonography to ascertain the feasibility of the pedicled nasoseptal flap after prior bilateral sphenoidotomy. Laryngoscope. 2010;120(9):1798-801.

15. Patel MR, Shah RN, Snyderman $\mathrm{CH}$, et al. Pericranial flap for endoscopic anterior skull-base reconstruction: clinical outcomes and radioanatomic analysis of preoperative planning. Neurosurgery. 2010;66(3):506-12 (discussion 512).

16. - Patel MR, Stadler ME, Snyderman $\mathrm{CH}$, et al. How to choose? Endoscopic skull base reconstructive options and limitations. Skull Base. 2010;20(6):397-404. Provides an algorithm for skull base reconstruction after endonasal endoscopic approaches.

17. •- Kassam AB, Prevedello DM, Carrau RL, et al. Endoscopic endonasal skull base surgery: analysis of complications in the 
authors' initial 800 patients. J Neurosurg. 2011;114(6): 1544-1568. Provides a detailed account of complication rates in one of the largest series in endonasal skull base surgery patients.

18. • Kono Y, Prevedello DM, Snyderman CH, et al. One thousand endoscopic skull base surgical procedures demystifying the infection potential: incidence and description of postoperative meningitis and brain abscesses. Infect Control Hosp Epidemiol. 2011;32(1):77-83. This article analyzes infectious complications in a significant series of endonasal skull base procedures and proves that these are not a higher risk for infection than open approaches.

19. - Pant H, Bhatki AM, Snyderman CH, et al. Quality of life following endonasal skull base surgery. Skull Base. 2010;20(1):35-40. This study demonstrates patient satisfaction and issues after endonasal surgery of the skull base. 